\title{
Monogenic Binary Pattern (MBP): A Novel Feature Extraction and Representation Model for Face Recognition
}

\author{
Meng Yang, Lei Zhang, Lin Zhang and David Zhang \\ Biometric Research Center, Dept. of Computing, The Hong Kong Polytechnic University \\ E-mail: \{csmyang, cslzhang, cslinzhang, csdzhang\}@comp.polyu.edu.hk
}

\begin{abstract}
A novel feature extraction method, namely monogenic binary pattern (MBP), is proposed in this paper based on the theory of monogenic signal analysis, and the histogram of MBP (HMBP) is subsequently presented for robust face representation and recognition. MBP consists of two parts: one is monogenic magnitude encoded via uniform LBP, and the other is monogenic orientation encoded as quadrant-bit codes. The HMBP is established by concatenating the histograms of MBP of all subregions. Compared with the well-known and powerful Gabor filtering based LBP schemes, one clear advantage of HMBP is its lower time and space complexity because monogenic signal analysis needs fewer convolutions and generates more compact feature vectors. The experimental results on the AR and FERET face databases validate that the proposed MBP algorithm has better performance than or comparable performance with state-of-the-art local feature based methods but with significantly lower time and space complexity.
\end{abstract}

\section{Introduction}

As one of the most active and visible research topics in computer vision, pattern recognition and biometrics, face recognition has been extensively studied in the past two decades [1], yet it is still a challenging problem in practice due to uncontrolled environments, occlusions and variations in pose, illumination, expression and aging, etc. Various methods have been proposed for face feature extraction, among which the representatives include Eigen-face [2], Fisher-face [3], Gabor Feature based Classification (GFC) [4] and LBP methods [5], etc.
Different from other face recognition methods, LBP methods use local structural information and histogram of sub-regions to extract and describe facial features. Following LBP, LGBPHS [6] was proposed to use Gabor filtering to enhance the facial features and then extract the local Gabor binary pattern histogram sequence, which improves much LBP's robustness to illumination changes. The Gabor phase was also used to improve the recognition rate [7-8], and a typical method of this class is the HGPP [8], which captures the Global Gabor phase and Local Gabor phase variation. Despite the high accuracy, the expense of the above mentioned Gabor filter based face recognition methods is also very expensive: both the computational cost and the storage space are high because Gabor filtering is usually applied at five different scales and along eight different orientations, which limits the application of these methods.

This paper presents a new local facial feature extraction method, namely monogenic binary pattern (MBP), based on the theory of monogenic signal analysis [9], and then proposes to use the histogram of MBP (HMBP) to describe the MBP features. Monogenic signal is a two-dimensional (2D) generalization of the one-dimensional analytic signal, through which the multi-resolution magnitude, orientation and phase of a 2D signal can be estimated. The proposed MBP combines monogenic orientation and monogenic magnitude information for face feature extraction and description. The advantage of MBP over other Gabor based methods [4][6][8] is that it has much lower time and space complexity but with better or comparable performance. This is mainly because monogenic signal analysis is itself a compact representation of features with little information loss. It does not use steerable filters to create multiorientation features like Gabor filters do. HMBP is the sub-region spatial histogram sequence of MBP features, which is robust to face image variation of 
lighting, pose, expression and occlusion, etc. The proposed MBP method is validated on the AR [10] and the FERET [11] face databases. The experimental results verified that $\mathrm{MBP}$ and $\mathrm{HMBP}$ are efficient and powerful local feature extractor and descriptor. They can achieve high face recognition rate but with low time and space complexity.

The rest of the paper is organized as follows. Section 2 presents in detail the MBP scheme for face recognition. Section 3 conducts experiments on the AR and FERET databases to evaluate the performance of the proposed method. Section 4 concludes the paper.

\section{MBP for face recognition}

\subsection{Monogenic representation of face image}

The monogenic signal analysis [9] is a framework to interpret images in terms of the local phase, the local orientation and the local magnitude. Different from obtaining Gabor magnitude, orientation and phase using steerable Gabor filters, the monogenic representation of $2 \mathrm{D}$ signals is accomplished via the Riesz transform [12]. Specifically, the local magnitude $A$, orientation $\theta \in[0 \pi$ ) and phase $\phi \in[02 \pi)$ of a $2 \mathrm{D}$ signal $I$ can be computed by [9]:

$$
\left\{\begin{array}{l}
A=\sqrt{h^{2}+h_{x}^{2}+h_{y}^{2}} \\
\theta=\operatorname{atan}\left(h_{y} / h_{x}\right) \\
\phi=-\operatorname{sign}\left(h_{x}\right) \operatorname{atan} 2\left(\sqrt{h_{y}^{2}+h_{x}^{2}} / h\right)
\end{array}\right.
$$

with

$$
\left\{\begin{array}{l}
h=I * F^{-1}(G(\omega)) \\
h_{d}=F^{-1}\left(\left(\sqrt{-1} \cdot \omega_{d} / \sqrt{\omega_{x}^{2}+\omega_{y}^{2}}\right) H\right), d \in\{x, y\}
\end{array}\right.
$$

where "** is the convolution operator, $G(\omega)$ is the Log-Gabor filter in Fourier domain, $F$ represents the 2D Fourier transform, $H=F(h)$ and $\omega_{x}$ and $\omega_{y}$ are the horizontal and vertical frequencies.

Since Log-Gabor filters are band-pass filters, usually multi-scale monogenic representation is required to fully describe a signal. Fig. 1 shows the monogenic representation of a face image at one scale. We can see that the facial local structures are well captured in its monogenic components.

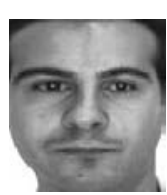

(a)

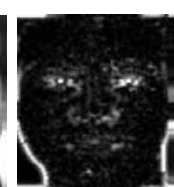

(b)

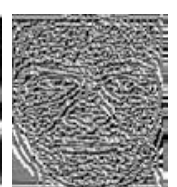

(c)

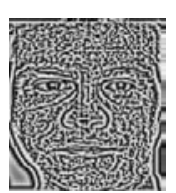

(d)
Fig. 1. A face image (a) and its monogenic representation at a scale: (b) magnitude component; (c) orientation component and (d) phase component.

\subsection{Monogenic binary pattern (MBP)}

In the monogenic representation of a face image, the magnitude $A$ is a measurement of local structure energy, and we can use the conventional LBP operator [13] to encode the variation of local energy. The LBP operator can encode a local $3 \times 3$ patch into an 8 -bits binary code. The uniform LBP operator was later proposed [14], which contains at most two bitwise transitions from 0 to 1 or vice versa when the binary string is considered circular. The uniform LBP uses 6bits to describe the local structure information without degrading much the performance of LBP. In our method, we use the uniform LBP to extract the local texture information in the monogenic magnitude component, and hence each pixel $Z$ in the magnitude code map, denoted by $B_{m}$, at each scale will be 6-bits.

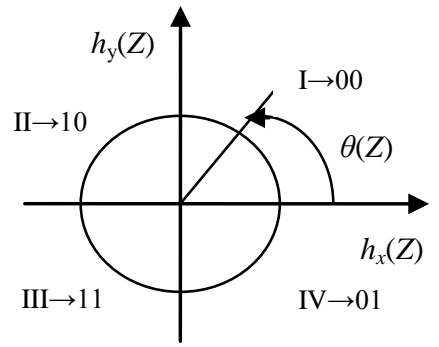

Fig. 2. Quadrant bit coding of monogenic orientation.

Apart from the magnitude, the monogenic orientation $\theta$ indicates the dominant direction of local image variation, which is an important feature in image recognition. Here we code the monogenic orientation information into a quadrant bit, which is illustrated in Fig. 2. A pixel $Z$ in the orientation map at each scale is encoded into two bits, $\left(B_{\theta}^{x}(Z), B_{\theta}^{y}(Z)\right)$, by the following rule:

$$
B_{\theta}^{d}(Z)=\left\{\begin{array}{ll}
0 & \text { if } h_{d}(Z)>0 \\
1 & \text { if } h_{d}(Z) \leq 0
\end{array}, \quad d \in\{x, y\}\right.
$$

where $h_{x}$ and $h_{y}$ (refer to Eq. (2)) are respectively the horizontal and vertical Riesz transform parts of monogenic signal representation.

The monogenic phase information is not used in the proposed MBP. How to effectively exploit and code the monogenic phase will be our future work.

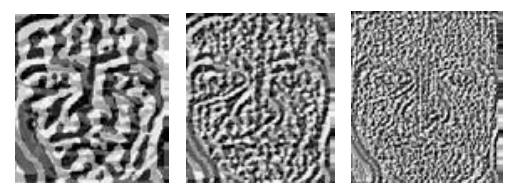

Fig. 3. The MBP maps of a face image at three scales. 
Finally, the proposed MBP is the combination of the binary codes of orientation and uniform LBP of magnitude, i.e. $\mathrm{MBP}=\left(B_{\theta}^{x}(Z), B_{\theta}^{y}(Z), B_{m}(Z)\right)$. It can be seen that the MBP at each scale is an 8-bits representation of the original face image. Fig. 3 shows the MBP maps of a face image at three scales.

\subsection{Histogram of MBP (HMBP)}

The statistical information of local image patches can be captured by local histograms, which are robust to the image occlusion and variations of pose, expression, and noise, etc. After obtaining the multiscale MBP maps of the face image, we construct the histogram of MBP (HMBP) through the following procedures. First, the MBP maps at each scale are divided into multiple non-overlapping regions. Then the local histogram is built from each sub-region. Finally, all the local histograms are concatenated into a single histogram vector to represent the face image. Formally, the HMBP feature is formulated as

$$
H M B P=\left(H_{M B P}(r, s), r=1, \cdots, L ; s=1,2, \cdots, S\right)
$$

where $L$ is the number of sub-regions at each scale; $S$ is the total scale of monogenic signal representation (in this work 3 scales are used); and $H_{M B P}(r, s)$ is the histogram of the $r^{\text {th }}$ sub-region at scale $s$.

\subsection{Face recognition by $\mathrm{HMBP}$}

In the paper, the similarity between two histograms $H_{1}$ and $H_{2}$ is defined as their intersection, which is computed as follows:

$$
S_{H I}\left(H_{1}, H_{2}\right)=\sum_{i=1}^{N} \min \left(H_{1}^{i}, H_{2}^{i}\right)
$$

where $N$ is the number of bins in the histogram, and $H_{1}^{i}$ and $H_{2}^{i}$ denote the frequency of the $i^{\text {th }}$ bin respectively in $H_{1}$ and $H_{2}$. As in [7-8], there are two variants to measure the similarity between HMBPs: the normal HMBP and the weighted HMBP, which can be both formulized as

$$
\begin{aligned}
& S(\text { HMBP', HMBP") } \\
& =\sum_{s=1}^{s} \sum_{r=1}^{L} w(r, s) S_{H I}\left(H_{M B P}^{\prime}(r, s), H_{M B P}^{\prime \prime}(r, s)\right)
\end{aligned}
$$

where $w(r, s)$ is the weight of the $r^{\text {th }}$ sub-region in the $s^{\text {th }}$ scale of HMBP. In the case of normal HMBP, all $w(r, s)$ equal to 1 , while in weighted HMBP, $w(r, s)$ varies considering that different sub-regions have different contributions to face recognition. The weights can be determined based on the Fisher criterion [8].

\section{Experimental results}

We test our algorithm on two benchmark face databases. One is the subset of AR database, consisting of over 4000 images of the frontal view faces from 126 subjects [10], and the other one is the FERET face database [11]. The state-of-the-art local feature based methods LBP [5], LGBPHS [6] and HGPP [8] are used for comparison (we thank the authors for sharing their codes). In the proposed approach, we empirically partitioned the face image into $8 \times 8$ sub-regions in the experiments.

\subsection{Time and space complexities}

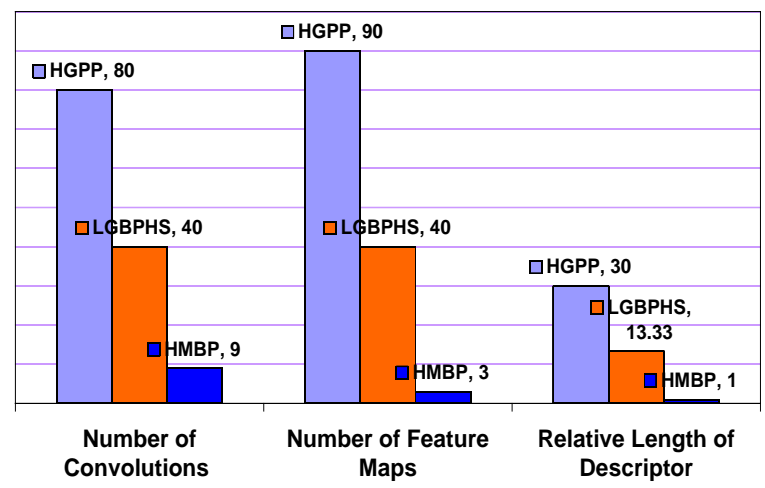

Fig. 4. Comparison of the time and space complexities among HGPP, LGBPHS and HMBP.

Let's first compare the time and space complexities between HMBP, LGBPHS and HGPP. In the feature histogram extraction, the convolution is the most timeconsuming processing. As shown in Fig.4, since HGPP and LGBPHS both use Gabor filtering for feature enhancement, they respectively need 40 and 80 Gabor filter convolutions per image. However, since monogenic signal representation does not use directional Gabor filtering, our method only needs 3 Log-Gabor filtering and 6 Riesz transforms per image. For space complexity, the HGPP produces 90 8-bits feature maps per image; LGBPHS produces 40 8-bits feature maps per image; while HMBP only produces 3 8-bits feature maps. That means that the length of HGPP and LGBPHS are about 30 and 13 times that of HMBP, and thus in similarity computing, HMBP needs much less time than HGPP and LGBPHS.

\subsection{Experiments on the AR database}

In the experiment, we used a subset of the AR database [10], consisting of 50 male subjects and 50 female subjects. In the case of no-occlusion, for each subject the seven images with illumination and expression changes from Session 1 were taken for training, and the other seven images with illumination and expression changes from Session 2 were used for testing. In the experiment of occlusion, only one neutral expression image of the first session was used to form the Gallery, and all the partially occluded 
images of the first session and the second session were used as the probes. The rank- 1 recognition rates of the experiments are listed in Table I. We can see that the proposed MBP outperforms LBP and LGBPHS. Its recognition rate is slightly less than HGPP. However, its time and space complexity is much less than HGPP, as we analyzed in section 3.1.

Table I. Rank -1 recognition rates by different methods on the AR database.

\begin{tabular}{|c|c|c|c|}
\hline \multirow{2}{*}{ Methods } & No occlusion & \multicolumn{2}{|c|}{ Occlusion } \\
\cline { 3 - 4 } & & sunglass & scarf \\
\hline HMBP & 98.57 & 92.0 & 91.50 \\
\hline HGPP & 99.0 & 93.0 & 92.33 \\
\hline LGBPHS & 97.71 & 91.0 & 89.0 \\
\hline LBP & 97.29 & 83.71 & 68.33 \\
\hline
\end{tabular}

\subsection{Experiments on the FERET database}

The FERET database [11] is often used to validate an algorithm's effectiveness because it contains many kinds of image variations. By taking fa subset as a gallery, the probe subsets fb and fc were captured with expression and illumination variations. Especially, Dup1 and Dup2 consist of images that were taken at different times with more than one year interval. The recognition results by different methods are shown in Table II, where the prefix " $W$ " means the weighted histogram similarity. From Table II, we can see again that the proposed MBP approaches have better performance than other methods except for HGPP. However, HGPP's time and space complexity is about 10 and 30 times than HLMBP. Therefore, the overall performance of MBP is better.

Table II. Rank -1 recognition rate by different methods on the FERET probe sets.

\begin{tabular}{|c|c|c|c|c|}
\hline \multirow{2}{*}{ Methods } & \multicolumn{4}{|c|}{ FERET probe sets } \\
\cline { 2 - 5 } & Fb & FC & Dup I & Dup II \\
\hline W-HMBP & 98.08 & 98.45 & 75.76 & 75.21 \\
\hline HMBP & 97.74 & 98.45 & 73.55 & 72.22 \\
\hline W-HGPP & 97.5 & 99.5 & 79.5 & 77.8 \\
\hline HGPP & 97.6 & 98.9 & 77.7 & 76.1 \\
\hline W-LGBPHS & 98.0 & 97.0 & 74.0 & 71.0 \\
\hline LGBPHS & 94.0 & 97.0 & 68.0 & 53.0 \\
\hline W-LBP & 97.0 & 79.0 & 66.0 & 64.0 \\
\hline LBP & 93.0 & 51.0 & 61.0 & 50.0 \\
\hline
\end{tabular}

\section{Conclusion}

We proposed a novel face representation model, namely monogenic binary model (MBP), based on the monogenic signal analysis. After monogenic signal transform, we coded the face image into 3 8-bits maps by using the magnitude component and the orientation component. Then the local histogram of MBP (HMBP) was used to represent the face image for matching.
Compared with the state-of-the-art local feature based methods LGBPHS and HGPP, the HMBP has significantly lower time and space complexities, yet the experimental results on AR and FERET databases showed that HMBP outperforms the LBP and LGBPHS methods and is only slightly worse than the HGPP method. In the future, we will investigate how to better exploit the monogenic signal representation for more efficient and robust face recognition.

\section{Acknowledgments}

This paper is supported by the Hong Kong RGC General Research Fund (PolyU5351/08E).

\section{References}

[1] W. Y. Zhao, R. Chellppa, P. J. Phillips, and A. Rosenfeld. Face recognition: A literature survey. ACM Computing Survey, pages 399-458, 2003.

[2] M. Turk and A. Pentland. Eigenfaces for recognition. Journal of Cognitive Neuroscience, 13(1):71-86, 1991.

[3] P. Belhumeur, J. Hespanha, and D. Kriegman. Egienfaces vs. fisherfaces: Recognition using class specific linear projection. IEEE TPAMI, 19(7):711-720, 1997.

[4] C. Liu and H. Wechsler. Gabor feature based classification using the enhanced Fisher linear discriminant model for face recognition. IEEE TPAMI, 11(4):467-476, 2002.

[5] A. Timo, H. Abdenour, and P. Matti. Face recognition with Local Binary Patterns. In Proc. ECCV , pages 469481, 2004.

[6] W. Zhang, S. Shan, W. Gao, X. Chen, and H. Zhang. Local Gabor binary pattern histogram sequence (LGBPHS): A novel non-statistical model for face representation and recognition. In Proc. ICCV, pages 786-791, 2005.

[7] W. Zhang, S. Shan, X. Chen, and W. Gao. Are Gabor Phases Really Useless for Face Recognition? In Proc. IC$P R$, pages 606-609, 2006.

[8] B. Zhang, S. Shan, X. Chen, and W. Gao. Histogram of Gabor Phase Patterns (HGPP): A Novel Object Representation Approach for Face Recognition. IEEE TIP, 16(1):57-68, 2006.

[9] M. Felsberg and G. Sommer. The monogenic signal. IEEE TSP, 49(12):3136-3144, 2001.

[10]A. M. Martinez and R. Benavente. The AR Face Database. CVC Technical Report \#24, June 1998.

[11]P. J. Phillips, H. M. Syed, A. Rizvi, and P. J. Rauss. The FERET evaluation methodology for face-recognition algorithms. IEEE TPAMI, 22(10):1090-1104, 2000.

[12]E. M. Stein and G. Weiss. Introduction to Fourier Analysis on Euclidean Spaces. Priceton, NJ: Princeton Univ. Press, 1971.

[13]T. Ojala, M. Pietikäinen, and D. Harwood. A comparative study of texture measures with classification based on feature distributions. PR 29:51-59, 199-6.

[14]T. Ojala, M. Pietikäinen, and T. Mäenpää. Multiresolution gray-scale and rotation invariant texture classification with local binary patterns. IEEE TPAMI, 24(7):971 -987, 2002. 